\title{
COMPLETENESS THEOREMS FOR UNIVERSAL AND IMPLICATIONAL LOGICS OF ALGEBRAS VIA CONGRUENCES
}

\author{
ROBERT W. QUACKENBUSH
}

(Communicated by Donald S. Passman)

\begin{abstract}
In this paper, simple algebraic proofs are given for the completeness theorems for the implicational and universal logics of algebras. The proofs are obtained by examining congruences, $\theta$, on the algebra of terms, $F(\omega)$, such that $F(\omega) / \theta$ belongs to the given class of algebras. Thus, they are direct analogs of $\mathrm{G}$. Birkhoff's proof of the completeness theorem for equational logic.
\end{abstract}

1. Introduction. Completeness theorems tend to depend on some version of the deduction theorem which is usually established by a tiresome induction based on lengths of derivations. An important exception is G. Birkhoff's completeness theorem for equational logic which uses the fundamental connection between equational theories and fully invariant congruences on the algebra of terms. In this paper I will show how to extend this connection to the logics associated with universal and implicational classes of algebras. The starting point is the observation that a universal class of algebras is determined by its countably generated members; the hope (which will be fulfilled) then is that universal and implicational theories can be identified by algebraic processes working on the algebra of terms, $\mathbf{F}(\omega)$ (i.e. the absolutely free algebra on a countable number of free generators). Indeed, we will see that implicational theories are in 1-1 correspondence with invariant algebraic closure operations on the congruence lattice of $\mathbf{F}(\omega)$. For universal theories the correspondence is more complicated while still being straightforward and natural.

I was attracted to this topic by a series of lectures by David Kelly in the universal algebra seminar at the University of Manitoba. He was lecturing on his paper [2], Complete rules of inference for universal sentences. During his presentation it occurred to me that at least for quasivarieties there ought to be a straightforward generalization of Birkhoff's theorem for varieties, and that the key to this generalization should be the existence of maximal quotients in quasivarieties. This turned out to be the case, and the details are little more than an exercise. In two ways Kelly's results are much more general than mine: he permits relations as well as operations, and he considers many more classes of models.

The presentation of this paper has been greatly improved by numerous helpful remarks of a referee to whom the author extends his appreciation.

Received by the editors January 30, 1987.

1980 Mathematics Subject Classification (1985 Revision). Primary 08C10; Secondary 08C15, $03 \mathrm{C} 60$.

Key words and phrases. Completeness theorem, quasivariety, universal class of algebras.

The research of the author was supported by a grant from the NSERC of Canada. 
2. Preliminaries. We will be dealing with universally quantified sentences of some fixed type containing no relation symbols; thus all our models are algebras. I will use Kelly's notation $S \rightarrow T$ for a universal sentence; here $S$ and $T$ are finite (possibly empty) sets of identities (i.e. atomic formulas) and $S \rightarrow T$ means that the conjunction of $S$ implies the disjunction of $T$. Either $S$ or $T$ may be empty; in case $S=\varnothing$ and $|T|=1, S \rightarrow T$ is an identity; if $S=T=\varnothing$, this is the universally false sentence, holding nowhere. For implicational logic we have $|T| \leq 1$. The classes of algebras I deal with all contain 1-element algebras. Now, $S \rightarrow T$ is satisfied in a 1-element algebra iff $T \neq \varnothing$. However, the completeness theorems will hold even for sets, $\Sigma$, of sentences containing a sentence of the form $S \rightarrow \varnothing$. This is because we will add a rule of inference which says that we infer $S^{\prime} \rightarrow \varnothing$ if we can derive $S \rightarrow \varnothing$ and if for every $\sigma \in S$, we can derive $S^{\prime} \rightarrow \sigma$. The universal algebraic or model theoretic results which I will be using are well known and are easily found in G. Grätzer [1].

3. Implicational logic. Recall that $S \rightarrow T$ is an implication if $|T| \leq 1$ and a strict implication if $|T|=1$; a class of algebras, $\mathbf{Q}$, is a quasivariety if it is defined by a set of strict implications. Given a set, $\Sigma$, of implications, $\operatorname{Mod} \Sigma$ is the class of all models of $\Sigma$; given a class, $\mathbf{K}$, of algebras, $\mathrm{Th}_{i} \mathbf{K}$ is the set of all implications true in every member of $\mathbf{K}$. The mapping $\Sigma \rightarrow \operatorname{Th}_{i} \operatorname{Mod} \Sigma$ is a closure operation; $\mathrm{Th}_{i} \operatorname{Mod} \Sigma$ is called an implicational theory. Note that elsewhere in the literature, implications are called universal Horn sentences (sometimes just Horn sentences) and strict implications are called quasi-identities.

For the completeness theorem for the implicational logic of algebras we will use the following important property of quasivarieties: given a quasivariety $\mathbf{Q}$ and an algebra $\mathbf{A}, \mathbf{Q}$ contains a maximal quotient $\mathbf{A}^{\prime}$ of $\mathbf{A}$ : let $f: \mathbf{A} \rightarrow \mathbf{A}^{\prime}$ be onto and $g: \mathbf{A} \rightarrow \mathbf{B}$ with $\mathbf{B}$ in $\mathbf{Q}$; then there is a unique $g^{\prime}: \mathbf{A}^{\prime} \rightarrow \mathbf{B}$ such that $g^{\prime} f=g$. Recall that $\mathbf{A}^{\prime}$ can be constructed as a subdirect product of all quotients of $\mathbf{A}$ in $\mathbf{Q}$; equivalently, Ker $f$ is the meet of all congruences, $\theta$, on $\mathbf{A}$ such that $\mathbf{A} / \theta \in \mathbf{Q}$.

Let $\mathbf{F}(\omega)$ be the countably generated absolutely free algebra of type $\tau$. We will use this maximal quotient property to construct a map from the compact congruences of $\mathbf{F}(\omega)$ to the congruences of $\mathbf{F}(\omega)$ by sending the compact congruence $\theta$ to the smallest congruence, $Q(\theta)$, of $\mathbf{F}(\omega)$ containing $\theta$ such that $\mathbf{F}(\omega) / Q(\theta) \in \mathbf{Q}$, i.e., $\mathbf{F}(\omega) / Q(\theta)$ is the maximal quotient of $\mathbf{F}(\omega) / \theta$ in $\mathbf{Q}$, and $Q$ is the maximal quotient map of $\mathbf{Q}$.

What does this construction have to do with implications? Consider $S \rightarrow(p=q)$ where $S=\left\{\left(p_{1}=q_{1}\right), \ldots,\left(p_{n}=q_{n}\right)\right\}$. Think of an identity $r=s$ as an ordered pair of elements from $\mathbf{F}(\omega)$. Thus, our implication becomes $S \rightarrow(p, q)$ where $S=\left\{\left(p_{1}, q_{1}\right), \ldots,\left(p_{n}, q_{n}\right)\right\}$; this notation will be used henceforth. Let $\theta(S)$ be the smallest congruence on $\mathbf{F}(\omega)$ containing $\left\{\left(p_{1}, q_{n}\right), \ldots,\left(p_{n}, q_{n}\right)\right\} ; \theta(S)$ is a compact congruence. Then $Q(\theta(S))$ is the smallest congruence containing $\theta(S)$ such that $\mathbf{F}(\omega) / Q(\theta(S)) \in \mathbf{Q}$. If $S \rightarrow(p, q)$ is true in $\mathbf{Q}$, then we must have $(p, q) \in Q(\theta(S))$.

LEMMA 1. The implication $S \rightarrow(p, q)$ holds in $\mathbf{Q}$ if and only if $(p, q) \in$ $Q(\theta(S))$.

PROOF. If $S \rightarrow(p, q)$ holds in $\mathbf{Q}$, then as $\left(p_{i}, q_{i}\right) \in Q(\theta(S))$ for $1 \leq i \leq n$, and as $\mathbf{F}(\omega) / Q(\theta(S)) \in \mathbf{Q}$, we must have $(p, q) \in Q(\theta(S))$. Conversely, if $S \rightarrow(p, q)$ fails in $\mathbf{Q}$, then it fails in some countably generated member, $\mathbf{A}$, of $\mathbf{Q}$. This means that 
there is an $\omega$-tuple, $\underline{a}$, of elements which generates $\mathbf{A}$ and such that $p_{i}(\underline{a})=q_{i}(\underline{a})$ for $1 \leq i \leq n$ while $p(\underline{a}) \neq q(\underline{a})$. But $p_{i}(\underline{a})=q_{i}(\underline{a})$ for $1 \leq i \leq n$ means that $\mathbf{A}$ is a quotient of $\mathbf{F}(\omega) / \theta(S)$. By the maximal quotient property, $\mathbf{A}$ is a quotient of $\mathbf{F}(\omega) / Q(\theta(S))$. Since $p(\underline{a}) \neq q(\underline{a}),(p, q) \notin Q(\theta(S))$.

THEOREM 2. Let $M$ be a map from the compact congruences of $\mathbf{F}(\omega)$ to the congruences of $\mathbf{F}(\omega) . M$ is a maximal quotient map for some quasivariety if and only if the following conditions hold:

(1) $\theta(S) \subseteq M(\theta(S))$ for each compact congruence $\theta(S)$.

(2) For every endomorphism, $f$, of $\mathbf{F}(\omega)$ and for each compact congruence, $\theta(S)$, $f(M(\theta(S))) \subseteq M(\theta(f(S)))$.

(3) For any compact congruences, $\theta(S)$ and $\theta(T)$, if $\theta(T) \subseteq M(\theta(S))$, then $M(\theta(T)) \subseteq M(\theta(S))$.

PROOF. Suppose that $Q$ is the maximal quotient map for the quasivariety $\mathbf{Q}$; (1) is explicit in the definition of $Q$. For (2), let $f$ be an endomorphism of $\mathbf{F}(\omega)$ and let $(p, q) \in Q(\theta(S))$; we must show that $(f(p), f(q)) \in Q(\theta(f(S)))$. By Lemma $1, S \rightarrow(p, q)$ holds in $\mathbf{Q}$ and hence in $\mathbf{F}(\omega) / Q(\theta(f(S)))$. But in $\mathbf{F}(\omega) / Q(\theta(f(S)))$ we have $f\left(p_{i}\right)=f\left(q_{i}\right)$ for $1 \leq i \leq n$; since $S \rightarrow(p, q)$ holds there, we must have $(f(p), f(q)) \in Q(\theta(f(S)))$. For $(3)$, since $\mathbf{F}(\omega) / Q(\theta(S)) \in \mathbf{Q}$ and $\theta(T) \subseteq Q(\theta(S))$, the definition of $Q(\theta(T))$ forces $Q(\theta(T)) \subseteq Q(\theta(S))$. Conversely, let $M$ be a map satisfying (1)-(3). We will prove that this map is the maximal quotient map of the quasivariety generated by all algebras $\mathbf{F}(\omega) / M(\theta(S))$ where $\theta(S)$ is a compact congruence on $\mathbf{F}(\omega)$. Thus, let $\mathbf{Q}$ be the quasivariety generated by all these algebras, and let $Q$ be the maximal quotient map of $\mathbf{Q}$. Trivially, $Q(\theta(S)) \subseteq M(\theta(S))$ for all $S$. Let $(p, q) \in M(\theta(S))$; we will show that $S \rightarrow(p, q)$ holds in each $\mathbf{F}(\omega) / M(\theta(T))$ and hence holds in $\mathbf{Q}$. By Lemma 1 , this will mean that $(p, q) \in Q(\theta(S))$, proving that $M(\theta(S))=Q(\theta(S))$ for all $S$. Let $\underline{a}$ be an $\omega$-tuple of elements from $\mathbf{F}(\omega)$, and let $\left(p_{i}(\underline{a}), q_{i}(\underline{a})\right) \in M(\theta(T))$ for $1 \leq i \leq n$; we must prove that $(p(\underline{a}), q(\underline{a})) \in$ $M(\theta(T))$. Let $f$ be the endomorphism of $\mathbf{F}(\omega)$ sending $x_{i}$ to $a_{i}$ where $x_{i}$ is the $i$ th free generator of $\mathbf{F}(\omega)$ and $a_{i}$ is the $i$ th component of $\underline{a}$. Thus, $\theta(f(S)) \subseteq M(\theta(T))$. By (3), $M(\theta(f(S)) \subseteq M(\theta(T))$, and by $(2), f(M(\theta(S))) \subseteq M(\theta(f(S)))$. Since $(p, q) \in M(\theta(S)),(p(\underline{a}), q(\underline{a})) \in M(\theta(T))$ as required.

It is now straightforward to use Lemma 1 and Theorem 2 to derive rules of inference for implicational logic; let $\Sigma$ be a set of implications. The rules are

(a) If $S \rightarrow T \in \Sigma$, then infer $S \rightarrow T$.

(b) For all $\sigma \in S$, infer $S \rightarrow \sigma$.

(c) For all $S$ and $p$, infer $S \rightarrow(p, p)$.

(d) From $S \rightarrow(p, q)$, infer $S \rightarrow(q, p)$.

(e) From $S \rightarrow(p, q)$ and $S \rightarrow(q, r)$ infer $S \rightarrow(p, r)$.

(f) From $S \rightarrow\left(p_{i}, q_{i}\right)$ for $1 \leq i \leq n$ and for each operation symbol, $r$, infer $S \rightarrow\left(r\left(p_{1}, \ldots, p_{n}\right), r\left(q_{1}, \ldots, q_{n}\right)\right)$.

(g) For every endomorphism, $f$, of $\mathbf{F}(\omega)$, from $S \rightarrow T$, infer $f(S) \rightarrow f(T)$.

(h) From $S \rightarrow \tau$ for all $\tau \in T$ and from $T \rightarrow U$, infer $S \rightarrow U$.

(i) For all $p$ and $q$, from $S \rightarrow \varnothing$, infer $S \rightarrow(p, q)$.

Rules (b)--(f) are obtained from (1): $M(\theta(S))$ is a congruence containing $\theta(S)$. Rule (g) is obtained from (2). As for (h), first let $U=\{v\}$. If $S \rightarrow \tau$ holds for all $\tau \in T$, then $T \subseteq M(\theta(S))$; by (3), $M(\theta(T)) \subseteq M(\theta(S))$. But as $v \in M(\theta(T))$, 
this means that $v \in M(\theta(S))$; i.e., we should infer $S \rightarrow U$. When $U=\varnothing$ we use rule (h) to derive $S \rightarrow \varnothing$ when $\Sigma$ does not consist entirely of strict implications. Also note that (a)-(i) are valid rules of inference for first order logic and hence for implicational logic.

THEOREM 3. The rules of inference (a)-(i) form a complete set of rules of inference for implicational theories of algebras.

Proof. We must show that if $S \rightarrow T \in \operatorname{Th}_{i} \operatorname{Mod} \Sigma$, then $S \rightarrow T$ can be derived from $\Sigma$ using rules (a)-(h). Let us first show that we can derive each member of $\mathrm{Th}_{i} \operatorname{Mod} \Sigma$ under the assumption that $\Sigma$ consists entirely of strict implications. For each $S$, let $M(\theta(S))$ be the set of all $(p, q)$ such that $S \rightarrow(p, q)$ can be derived from $\Sigma$ by rules (a)-(i). Our rules guarantee that the mapping, $M$, is a maximal quotient map. Hence, by Lemma 1 and Theorem $3,\{S \rightarrow(p, q) \mid(p, q) \in M(\theta(S))\}$ is an implicational theory containing $\Sigma$ and hence containing $\operatorname{Th}_{i} \operatorname{Mod} \Sigma$, the smallest implicational theory containing $\Sigma$. This proves that every $S \rightarrow(p, q) \in \operatorname{Th}_{i} \operatorname{Mod} \Sigma$ can be derived using rules (a)-(i). Now suppose that $\Sigma$ contains a sentence of the form $U \rightarrow \varnothing$. Replace every sentence in $\Sigma$ of the form $U \rightarrow \varnothing$ with $U \rightarrow(x, y)$ where $x$ and $y$ are variables not occurring in $U$; this yields $\Sigma^{\prime}$, a set of strict implications. The nontrivial models of $\Sigma$ and $\Sigma^{\prime}$ coincide: clearly, a model of $\Sigma$ is a model of $\Sigma^{\prime}$; if $U \rightarrow \varnothing \in \Sigma$, then a model of $\Sigma^{\prime}$ failing $U \rightarrow \varnothing$ satisfies $U \rightarrow(p, q)$ for every $p$ and $q$ and so is trivial. Suppose that $V \rightarrow \varnothing \in \operatorname{Th}_{i} \operatorname{Mod} \Sigma$; thus $V \rightarrow(p, q) \in \operatorname{Th}_{i} \operatorname{Mod} \Sigma$ for all $p$ and $q$, and so $V \rightarrow(p, q) \in \operatorname{Th}_{i} \operatorname{Mod} \Sigma^{\prime}$. But as $\Sigma^{\prime}$ consists of strict implications, we know that we may derive $V \rightarrow(p, q)$ for any $p$ and $q$. Since $\Sigma$ contains a nonstrict implication, $U \rightarrow \varnothing$, we may derive $V \rightarrow \varnothing$ using rules (a) and (h).

The first proof of the completeness theorem for implicational classes of algebras is due to A. Selman [3]; that proof is entirely different from the one just presented (see [2] for further references).

4. Universal logic. Let $\mathbf{K}$ be a universal class of algebras defined by a set, $\Sigma$, of universal sentences; let $\mathbf{K}$ contain a 1-element algebra. This means that if $S \rightarrow T$ is true in $\mathbf{K}$, then $T \neq \varnothing$. Define $\Theta(S, T)$ to be $\{(p, q) \mid S \rightarrow T \cup(p, q)$ is true in $\mathbf{K}$ \}.

LEMMA 4. $\Theta(S, T)$ has the following properties:

(1) If $S \rightarrow T \cup(p, q) \in \Sigma$, then $(p, q) \in \Theta(S, T)$.

(2) $\Theta(S, T)$ is a congruence on $\mathbf{F}(\omega)$ containing $\theta(S)$.

(3) If $S \subseteq S^{\prime}$ and $T \subseteq T^{\prime}$, then $\Theta(S, T) \subseteq \Theta\left(S^{\prime}, T^{\prime}\right)$.

(4) If $f$ is an endomorphism of $\mathbf{F}(\omega)$, then $f(\Theta(S, T)) \subseteq \Theta(f(S), f(T))$.

(5) If $(r, s) \in \Theta(S, T \cup(p, q))$, then $(p, q) \in \Theta(S, T \cup(r, s))$.

(6) $\Theta(S \cup(p, q), T) \cap \Theta(S, T \cup(p, q))=\Theta(S, T)$.

PROOF. Properties (1)-(4) are clear. Property (5) is an acknowledgement of the asymmetry of $\Theta(S, T)$ vis-a-vis $S \rightarrow T \cup(p, q)$. For property $(6), \Theta(S, T) \subseteq$ $\Theta(S \cup(p, q), T) \cap \Theta(S, T \cup(p, q))$ by (3). For the other $\operatorname{direction}$, let $\mathbf{A}$ be any member of $\mathbf{K}$ and assume that $S \cup(p, q) \rightarrow T \cup(r, s)$ and $S \rightarrow T \cup(p, q) \cup(r, s)$ hold in $\mathbf{A}$. Then it is straightforward to check that $S \rightarrow T \cup(r, s)$ holds in $\mathbf{A}$.

Our congruence $\Theta(S, T)$ is analogous to $Q(\theta(S))$, but is not defined algebraically as $Q(\theta(S))$ was; how do we relate it to $\mathbf{K}$ ? An obvious relation would be that 
$\mathbf{F}(\omega) / \Theta(S, T) \in \mathbf{K}$; however, this need not be true. The connection with $\mathbf{K}$ is much more tenuous. Since $\mathbf{K}$ contains a 1-element algebra, we have that $S \rightarrow T$ holds in $\mathbf{K}$ iff $T \neq \varnothing$ and $\Theta(S, T)=\iota$ ( $\iota$ is the largest congruence on an algebra). The connection with $\mathbf{K}$ is made via the set, $P(\mathbf{K})$, of all $(S, T)$ such that $S \rightarrow T$ does not hold in $\mathbf{K}$; note that $(S, T) \in P(\mathbf{K})$ iff $T=\varnothing$ or if $\Theta(S, T)<\iota . P(\mathbf{K})$ is ordered by containment $(S, T) \leq\left(S^{\prime}, T^{\prime}\right)$ if and only if $S \subseteq S^{\prime}$ and $T \subseteq T^{\prime}$; note that if $S \subseteq S^{\prime}, T \subseteq T^{\prime}$ and $\left(S^{\prime}, T^{\prime}\right) \in P(\mathbf{K})$, then $(S, T) \in P(\mathbf{K})$.

Recalling that $\mathbf{K}$ is determined by its countably generated members, let us choose a congruence $\phi$ on $\mathbf{F}(\omega)$ such that $\mathbf{F}(\omega) / \phi \in \mathbf{K}$. Now choose finite subsets $S, T$ of $(\mathbf{F}(\omega))^{2}$ such that $S \subseteq \phi$ and $T \cap \phi=\varnothing$. Then $S \rightarrow T$ does not hold in $\mathbf{K}$ as it does not hold in $\mathbf{F}(\omega) / \phi$. Let us define $\mathbf{I}(\phi)=\{(S, T)|| S \cup T \mid<\omega, S \subseteq \phi$ and $T \subseteq(\iota-\phi)\} ; \mathbf{I}(\phi)$ is a subset of $P(\mathbf{K})$. Note that $\phi=\{(p, q) \mid((p, q), \varnothing) \in \mathbf{I}(\phi)\}$.

LEMMA 5. I $(\phi)$ has the following properties:

(a) $\mathbf{I}(\phi)$ is an order ideal of $P(\mathbf{K}):$ if $(S, T) \leq\left(S^{\prime}, T^{\prime}\right) \in \mathbf{I}(\phi)$, then $(S, T) \in \mathbf{I}(\phi)$.

(b) $\mathbf{I}(\phi)$ is directed: if $(S, T),\left(S^{\prime}, T^{\prime}\right) \in \mathbf{I}(\phi)$, then $\left(S \cup S^{\prime}, T \cup T^{\prime}\right) \in \mathbf{I}(\phi)$.

(c) For each $(p, q) \in(\mathbf{F}(\omega))^{2}$, either $((p, q), \varnothing) \in \mathbf{I}(\phi)$ or $(\varnothing,(p, q)) \in \mathbf{I}(\phi)$.

The proof of Lemma 5 is easy and is omitted. A subset $\mathbf{I}$ of $P(\mathbf{K})$ will be called a prime order ideal if I satisfies properties (a)-(c) of Lemma 5 . The connection between the mapping $(S, T) \rightarrow \Theta(S, T)$ and $\mathbf{K}$ is that the prime order ideals of $P(\mathbf{K})$ are in 1-1 correspondence with the congruences $\phi$ on $\mathbf{F}(\omega)$ such that $\mathbf{F}(\omega) / \phi \in$ $\mathbf{K}$.

THEOREM 6. Let $\mathbf{I}$ be a prime order ideal of $P(\mathbf{K})$ and define $\Psi(\mathbf{I})$ to be $\{(p, q) \mid((p, q), \varnothing) \in \mathbf{I}\}$. Then $\Psi(\mathbf{I})$ is a congruence on $\mathbf{F}(\omega)$ such that $\mathbf{F}(\omega) / \Psi(\mathbf{I}) \in$ $\mathbf{K}$.

ProOF. Since $\varnothing \rightarrow(p, p)$ is true in $\mathbf{K},(\varnothing,(p, p)) \notin P(\mathbf{K})$, and so $((p, p), \varnothing)$ is in $\mathbf{I}$ for all $p \in \mathbf{F}(\omega)$, i.e. $\Psi(\mathbf{I})$ is reflexive. Suppose $(p, q) \in \Psi(\mathbf{I})$. If $(\varnothing,(q, p))$ were in $\mathbf{I}$, then we would have $((p, q),(q, p)) \in \mathbf{I}$. But $(p, q) \rightarrow(q, p)$ is true in $\mathbf{K}$, so $((p, q),(q, p))$ is not in $P(\mathbf{K})$. Hence, $(\varnothing,(q, p)) \notin \mathbf{I}$ so that $(q, p) \in \Psi(\mathbf{I})$, making $\Psi(\mathbf{I})$ symmetric. In a similar manner, $\Psi(\mathbf{I})$ is transitive and has the substitution property; thus, $\Psi(\mathbf{I})$ is a congruence on $\mathbf{F}(\omega)$. To see that $\mathbf{F}(\omega) / \Psi(\mathbf{I}) \in \mathbf{K}$, let $S \rightarrow T$ hold in $\mathbf{K}$; we must show that it holds in $\mathbf{F}(\omega) / \Psi(\mathbf{I})$. Since $\mathbf{K}$ contains a 1-element algebra, $T \neq \varnothing . S \rightarrow T$ holds in $\mathbf{F}(\omega) / \Psi(\mathbf{I})$ iff for every endomorphism $f$ of $\mathbf{F}(\omega)$, if $f(S) \subseteq \Psi(\mathbf{I})$, then $f(T) \cap \Psi(\mathbf{I}) \neq \varnothing$. But $S \rightarrow T$ true in $\mathbf{K}$ means that $f(S) \rightarrow f(T)$ is true in $\mathbf{K}$; in particular, $(f(S), f(T)) \notin \mathbf{I}$. But if $f(S) \subseteq \Psi(\mathbf{I})$ and $f(T) \cap \Psi(\mathbf{I})=\varnothing$, then $(\varnothing, f(T))$ and $(f(S), \varnothing)$ are in I, forcing $(f(S), f(T))$ to be in I. This contradiction forces $S \rightarrow T$ to be true in $\mathbf{F}(\omega) / \Psi(\mathbf{I})$.

Notice that for every $(S, T) \in P(\mathbf{K})$ there is a prime order ideal of $P(\mathbf{K})$ containing $(S, T): S \rightarrow T$ fails in $\mathbf{K}$ and so fails in some quotient of $\mathbf{F}(\omega)$ belonging to $\mathbf{K}$. We are now ready for our abstract characterization of universal theories in terms of $\mathbf{F}(\omega)$. Let $\Gamma=\left\{(S, T) \mid S, T\right.$ are finite subsets of $\left.(\mathbf{F}(\omega))^{2}\right\} ; \Gamma$ is ordered in the obvious way. Let $\Phi$ be a map from $\Gamma$ to the congruence lattice of $\mathbf{F}(\omega)$ such that:

(1) $\Phi(\varnothing, \varnothing)<\iota$.

(2) $\Phi(S, T)$ is a congruence on $\mathbf{F}(\omega)$ containing $S$.

(3) If $(S, T) \leq\left(S^{\prime}, T^{\prime}\right)$, then $\Phi(S, T) \subseteq \Phi\left(S^{\prime}, T^{\prime}\right)$. 
(4) If $f$ is an endomorphism of $\mathbf{F}(\omega)$, then $f(\Phi(S, T)) \subseteq \Phi(f(S), f(T))$.

(5) If $(r, s) \in \Phi(S, T \cup(p, q))$, then $(p, q) \in \Phi(S, T \cup(r, s))$.

(6) $\Phi(S, T \cup(p, q)) \cap \Psi(S \cup(p, q), T)=\Phi(S, T)$.

Next, define $P(\Phi)$ to be $\{(S, T) \in \Gamma \mid T=\varnothing$ or $\Phi(S, T)<\iota\}$. By $(3), P(\Phi)$ is an order ideal of $\Gamma$. As above, a prime order ideal, $\mathbf{I}$, of $P(\Phi)$ is an order ideal which is directed and such that for all $(p, q) \in \mathbf{F}(\omega)$ either $((p, q), \varnothing) \in \mathbf{I}$ or $(\varnothing,(p, q)) \in \mathbf{I}$. For a prime order ideal, I, let $\Delta(\mathbf{I})$ be $\{(p, q) \mid((p, q), \varnothing) \in \mathbf{I}\}$.

LEMMA 7. $\Delta(\mathbf{I})$ is a congruence on $\mathbf{F}(\omega)$.

ProOF. Reflexivity: by $(2),(p, p) \in \Phi(\varnothing,(r, s))$ and so by property $(5),(r, s) \in$ $\Phi(\varnothing,(p, p))$. Thus, $\Phi(\varnothing,(p, p))=\iota$, implying that $(\varnothing,(p, p))$ is not in I and therefore that $((p, p), \varnothing)$ is in $\mathbf{I}$; i.e. $\Delta(\mathbf{I})$ is reflexive. Symmetry: let $(p, q) \in \mathbf{I}$; we will show that $\Phi((p, q),(q, p))=\iota$, implying that $(q, p) \in \mathbf{I}$. By $(2),(q, p) \in$ $\Phi((p, q),(r, s))$, and so by $(5),(r, s)$ is in $\Phi((p, q),(q, p))$. Transitivity and substitution are proved in a similar manner.

LEMMA 8. Every element of $P(\Phi)$ is contained in some prime order ideal of $P(\Phi)$.

PrOOF. The order ideal of $P(\Phi)$ generated by any element of $P(\Phi)$ is directed. Thus by Zorn's lemma, it suffices to show that for every directed order ideal $\mathbf{J}$ of $P(\Phi)$ and every $p, q \in \mathbf{F}(\omega)$, there is a directed order ideal I containing $\mathbf{J}$ and containing either $((p, q), \varnothing)$ or $(\varnothing,(p, q))$. If there were no such $\mathbf{I}$, then there would be $(S, T)$ and $\left(S^{\prime}, T^{\prime}\right)$ in $\mathbf{J}$ such that $(S \cup(p, q), T) \notin P(\Phi)$ and $\left(S^{\prime}, T^{\prime} \cup(p, q)\right) \notin$ $P(\Phi)$. Since $\mathbf{J}$ is directed, $\left(S^{\prime \prime}, T^{\prime \prime}\right)=\left(S \cup S^{\prime}, T \cup T^{\prime}\right) \in \mathbf{J}$. Thus, $\Phi\left(S^{\prime \prime} \cup(p, q), T^{\prime \prime}\right)=$ $\iota=\Phi\left(S^{\prime \prime}, T^{\prime \prime} \cup(p, q)\right)$. But then by $(6), \Phi\left(S^{\prime \prime}, T^{\prime \prime}\right)=\iota$, contradicting $\left(S^{\prime \prime}, T^{\prime \prime}\right) \in \mathbf{J}$.

Let $\mathbf{K}$ be the universal class generated by $\{\mathbf{F}(\omega) / \Delta(\mathbf{I}) \mid \mathbf{I}$ is a prime order ideal of $P(\Phi)\}$. For the universal sentence $S \rightarrow T$, recall that $\Theta(S, T)$ was defined to be $\{(p, q) \mid S \rightarrow T$ is true in $\mathbf{K}\}$.

THEOREM 9. $\Theta(S, T)=\Phi(S, T)$ for all $(S, T) \in \Gamma$.

ProOf. Let $(p, q) \in \Theta(S, T)$. By $(2),(p, q) \in \Theta(S \cup(p, q), T)$. If $(p, q) \in$ $\Phi(S, T \cup(p, q))$, then by $(6),(p, q) \in \Phi(S, T)$. If $(p, q) \notin \Phi(S, T \cup(p, q))$, then $(S, T \cup(p, q)) \in P(\Phi)$ and so there is a prime order ideal I containing $(S, T \cup$ $(p, q))$. But then $S \rightarrow T \cup(p, q)$ fails in $\mathbf{F}(\omega) / \Delta(\mathbf{I})$, contradicting $(p, q) \in \Theta(S, T)$. Conversely, let $(p, q) \in \Phi(S, T)$. We must prove that for every prime order ideal I of $P(\Phi), S \rightarrow T \cup(p, q)$ holds in $\mathbf{F}(\omega) / \Delta(\mathbf{I})$. Thus, let $f$ be an endomorphism of $\mathbf{F}(\omega)$ such that $f(S) \subseteq \Delta(\mathbf{I})$ and $f(T) \cap \Delta(\mathbf{I})=\varnothing$. This means that $(f(S), f(T)) \in$ I. We must prove that $(f(p), f(q)) \in \Delta(\mathbf{I})$; i.e. that $(f(p), f(q)) \in \mathbf{I}$. By (4), $(p, q) \in \Phi(S, T)$ implies that $(f(p), f(q)) \in \Phi(f(S), f(T))$. By $(3),(f(p), f(q)) \in$ $\Phi(f(S), f(T) \cup(r, s))$ and so by $(5),(r, s) \in \Phi(f(S), f(T) \cup(f(p), f(q)))$ for all $(r, s)$. That is, $\Phi(f(S), f(T) \cup(f(p), f(q)))=\iota$ so that $(f(S), f(T)) \cup(f(p), f(q))) \notin \mathbf{I}$. As $(f(S), f(T)) \in \mathbf{I}$ and $\mathbf{I}$ is directed, this means that $(\varnothing,(f(p), f(q)) \notin \mathbf{I}$. Therefore, $((f(p), f(q)), \varnothing) \in \mathbf{I}$ and $(f(p), f(q)) \in \Delta(\mathbf{I})$ as required.

We are now ready to translate conditions (1)-(6) into rules of inference. We let $\Sigma$ be a set of universal sentences. Our rules of inference are (a)-(i) from the last section and the following new rules.

(j) From $S \rightarrow T \cup(p, q)$ and for $S \subseteq S^{\prime}$ and $T \subseteq T^{\prime}$, infer $S^{\prime} \rightarrow T^{\prime} \cup(p, q)$.

(k) From $S \rightarrow T \cup(p, q) \cup(r, s)$ and $S \cup(p, q) \rightarrow T \cup(r, s)$, infer $S \rightarrow T \cup(r, s)$. 
Notice that property (5) is not translated into a rule of inference as it is covered by our tacitly assumed rules of inference (e.g., that in $S \rightarrow T, S$ is a set of identities rather than an explicitly listed and parenthesized conjunction of identities). Also note that $(\mathrm{j})$ and $(\mathrm{k})$ are valid rules of inference.

THEOREM 11. The rules (a)-(k) form a complete set of rules of inference for universal theories of algebras.

The proof of Theorem 11 is similar to that of Theorem 4 and is omitted. The completeness theorem for universal classes (where relations as well as operations are permitted) is due to D. Kelly [2].

\section{REFERENCES}

1. G. Grätzer, Universal algebra, 2nd ed., Springer-Verlag, Berlin and New York, 1979.

2. D. Kelly, Complete rules of inference for universal sentences, Studia Sci. Math. Hungar. 19 (1984), 347-361.

3. A. Selman, Completeness of calculi for axiomatically defined classes of algebras, Algebra Universalis 2 (1972), 20-32.

Department of MAThematics, University of MANitoba, WinNipeg, Manitoba, CANADA R3T 2N2 